\title{
EDITORIAL
}

\section{Closing the barn door...?}

\author{
The decision of an independent oversight committee to recommend the redaction of sensitive \\ information from two influenza research papers highlights the complexities of dual-use research.
}

Instinctively, most scientists rigorously defend the need for openness in scientific research. The scientific method depends on the ability of researchers to repeat each other's work, and requests for sharing of materials are usually granted. However, for researchers engaged in dual-use research - that is, biological research that could potentially be misused for nefarious purposes things are not always so simple. The complex issues surrounding the practice and publication of such research have been thrown into stark relief lately by the growing controversy surrounding two influenza papers.

At the time of going to press, the papers were under consideration at Science and Nature and were reported to describe the creation of a highly pathogenic $\mathrm{H} 5 \mathrm{~N} 1$ influenza virus that is capable of airborne transmission in ferrets. The US National Science Advisory Board for Biosecurity (NSABB) was asked to review the papers and, in a highly unusual move, has recommended that the 'experimental details and mutation data that would enable replication of the experiments' be removed, although the general conclusions of the manuscripts can be published. Should the redaction go ahead, then both journals are believed to have insisted, rightly, that the redacted information be made available to 'genuine' researchers, but exactly what form this access will take, and how it will be regulated, is still unclear. In addition, the NSABB requested that the manuscripts be modified in order to better explain the aims of the research and its potential public health benefits, and to detail the safety measures taken to protect researchers and the public.

Requesting such additional emphasis is not new. In 2005, genome sequencing was completed for the H1N1 influenza virus responsible for the 1918 Spanish flu pandemic that killed 20-50 million people worldwide $^{1}$; the virus was then reconstructed using reverse genetics, and its pathogenicity was studied in a mouse model of infection ${ }^{2}$. The NSABB also reviewed those papers, but on that occasion they supported publication and requested only the inclusion of a short statement to emphasize the protective precautions taken and the intentions of the research. At that time, there was a similar furore in the scientific and mainstream media, with some commentators suggesting that the risk of the reconstructed virus escaping was "almost an inevitability” (REF. 3). No such escape has occurred, and work on the reconstructed virus has helped us understand exactly why this virus was so devastating.

The request for redaction is therefore unprecedented and has provoked a storm of protest and comment from both sides, with some accusing the research groups involved of irresponsibility for conducting the research in the first place, and others lamenting the idea of censorship in any form. Why has this research prompted this unusual step, and is it justified? Full details of the experiments are not available as the papers have yet to be published, but there are two obvious elements that lead to concern. One is the model system involved. This work was carried out in ferrets, which are generally acknowledged as the best available small-animal model for influenza, as they recapitulate many of the clinical signs and symptoms of human infection and allow both pathogenicity and transmissibility to be studied. Thus, although it is impossible to know how this virus would behave in humans, the ferret model provides researchers with the best approximation. The other element, of course, is the virus itself. This work involves a highly pathogenic $\mathrm{H} 5 \mathrm{~N} 1$ virus. Since its re-emergence in 2003, almost 600 cases of $\mathrm{H} 5 \mathrm{~N} 1$ influenza have been reported in humans, and more than 300 have been fatal. This 'mortality rate' - more than $50 \%$ - is $25 \%$ greater than that of the 1918 virus. However, as respected virologist Vincent Racianello points out on his blog, recent research ${ }^{4,5}$ has indicated that there could be a substantial number of subclinical H5N1 infections; if true, this means that the mortality rate of the virus may actually be much lower.

Undoubtedly, in weighing up these and many other considerations, the NSABB, which includes many active microbiologists among its membership, did not take the decision lightly. But it is unclear whether the benefits of the redaction outweigh the risks of publication. As many microbiologists have pointed out, given that the results of one study have already been presented at a conference and widely reported, sufficient information maybe already in the public domain for this to be a case of closing the barn door after the horse has bolted.

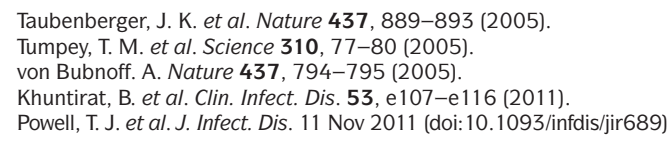

